\title{
Genetic and clinical specificity of 26 symptomatic carriers for dystrophinopathies at pediatric age
}

\section{This article has been corrected since online publication and a corrigendum is also printed in this issue}

\author{
Sandra Mercier ${ }^{1,2}$, Annick Toutain ${ }^{3}$, Aurélie Toussaint ${ }^{2}$, Martine Raynaud $^{3}$, Claire de Barace ${ }^{4}$, \\ Pascale Marcorelles ${ }^{5}$, Laurent Pasquier ${ }^{6}$, Martine Blayau ${ }^{7}$, Isabelle Pénisson-Besnier ${ }^{8}$, Norma Romero ${ }^{9}$, \\ Caroline Espil ${ }^{10}$, Philippe Parent ${ }^{11}$, Hubert Journel ${ }^{12}$, Leila Lazaro ${ }^{13}$, Jon Andoni Urtizberea ${ }^{14}$, \\ Alexandre Moerman ${ }^{15}$, Laurence Faivre ${ }^{16}$, Bruno Eymard ${ }^{17}$, Kim Maincent ${ }^{18}$, Romain Gherardi ${ }^{19}$, \\ Denys Chaigne ${ }^{20}$, Rabah Ben Yaou ${ }^{2}$, France Leturcq ${ }^{2}$, Jamel Chelly ${ }^{2}$ and Isabelle Desguerre ${ }^{\star 2,21}$
}

The molecular basis underlying the clinical variability in symptomatic Duchenne muscular dystrophy (DMD) carriers are still to be precised. We report 26 cases of early symptomatic DMD carriers followed in the French neuromuscular network. Clinical presentation, muscular histological analysis and type of gene mutation, as well as $\mathrm{X}$-chromosome inactivation (XCl) patterns using DNA extracted from peripheral blood or muscle are detailed. The initial symptoms were significant weakness $(88 \%)$ or exercise intolerance (27\%). Clinical severity varied from a Duchenne-like progression to a very mild Becker-like phenotype. Cardiac dysfunction was present in $19 \%$ of the cases. Cognitive impairment was worthy of notice, as $27 \%$ of the carriers are concerned. The muscular analysis was always contributive, revealing muscular dystrophy (83\%), mosaic in immunostaining $(81 \%)$ and dystrophin abnormalities in western blot analysis (84\%). In all, $73 \%$ had exonic deletions or duplications and $27 \%$ had point mutations. $\mathrm{XCI}$ pattern was biased in $62 \%$ of the cases. In conclusion, we report the largest series of manifesting DMD carriers at pediatric age and show that exercise intolerance and cognitive impairment may reveal symptomatic DMD carriers. The complete histological and immunohistological study of the muscle is the key of the diagnosis leading to the dystrophin gene analysis. Our study shows also that cognitive impairment in symptomatic DMD carriers is associated with mutations in the distal part of the $D M D$ gene. $\mathrm{XCl}$ study does not fully explain the mechanisms as well as the wide spectrum of clinical phenotype, though a clear correlation between the severity of the phenotype and inactivation bias was observed. European Journal of Human Genetics (2013) 21, 855-863; doi:10.1038/ejhg.2012.269; published online 9 January 2013

Keywords: dystrophin; female carrier; $\mathrm{X}$ inactivation

\section{INTRODUCTION}

Duchenne muscular dystrophy (DMD) has always been extensively described in its clinical presentation, evolution and severity. ${ }^{1}$ However, recent studies pointed out that the same mutation can be responsible for DMD phenotypes of different severity suggesting involvement of modifier and/or epigenetic factors. ${ }^{2,3}$ It has been estimated that about $8 \%$ of DMD female carriers have some manifestations including cardiomyopathy and/or some degree of weakness that could be highlighted by careful clinical examination..$^{4-8}$ Relationships between clinical phenotype and dystrophin abnormalities in muscle tissue among female carriers of DMD gene mutations were previously investigated. ${ }^{9}$ However, a comprehensive view of factors underlying clinical symptoms occurrence and severity is still lacking.
$D M D$ gene is very large (more than $2.5 \mathrm{Mb}$ ) and therefore difficult to test especially in females. Development of multiplex PCR method has allowed identification of about $95 \%$ of all deletions by screening of only 19 exons. However, this qualitative approach cannot be used to identify duplications or to determine female status for deletions and duplications. Currently, other diagnostic approaches, such as semi-quantitative fluorescent PCR (MLPA) and use of CGHgene-specific array represent reliable alternatives. ${ }^{10}$

The widely proposed explanation for the occurrence of clinical manifestations in heterozygous females is preferential skewed inactivation of the $\mathrm{X}$ chromosome bearing the non-mutated $D M D$ allele. ${ }^{11-16}$ Female carriers with manifesting muscle weakness usually have a mosaic expression of dystrophin in muscle shown by immunostaining, but the

\footnotetext{
${ }^{1}$ Department of Medical Genetics, CHU Nantes, France; ${ }^{2}$ Paris V University, Institut Cochin, INSERM U1016, Paris, France; ${ }^{3}$ Department of Genetics, CHU Clocheville, Tours, France; ${ }^{4}$ Department of Pediatry, Hôpital Yves Le Foll, Saint Brieuc, France; ${ }^{5}$ Department of Neuropathology, CHU Brest, Brest, France; ${ }^{6}$ Department of Medical Genetics, CLAD Ouest, CHU Rennes, Rennes, France; ${ }^{7}$ Department of Molecular Genetics, CHU Pontchaillou, Rennes, France; ${ }^{8}$ Department of Neurology, CHU Angers, Angers, France; ${ }^{9}$ Institute of Myology, CHU Hôpital Pitié-Salpétrière, Paris, France; ${ }^{10}$ Department of Neuropediatry, CHU Pellerin, Bordeaux, France; ${ }^{11}$ Department of Genetics, CHU Morvan, Brest, France; ${ }^{12}$ Department of Genetics, CH Vannes, Vannes, France; ${ }^{13}$ Department of Pediatry, CH Côte Basque, Bayonne, France; ${ }^{14} \mathrm{APHP}$, Centre de référence maladies neuromusculaires, Hendaye, France; ${ }^{15}$ Department of Genetics, CHU Jeanne de Flandres, Lille, France; ${ }^{16}$ Department of Genetics, CHU Dijon, Dijon, France; ${ }^{17}$ APHP, Department of Neuromuscular disorders, CHU Salpétrière, Paris, France; ${ }^{18}$ APHP, Department of Neuropediatry, CHU Trousseau, Paris, France; ${ }^{19}$ APHP, Department of Histology, INSERM U841, CHU Mondor, Creteil, France; ${ }^{20}$ Department of Pediatry, CHU Strasbourg, Strasbourg, France; ${ }^{21}$ APHP, Department of Neuropediatry, CHU Necker, Paris, France

*Correspondence: Professor I Desguerre, APHP, Department of Neuropediatry, Hôpital Necker, 149 rue de Sèvres, Paris, 75015 , France. Tel: + 330 014 4494856 ; Fax: + 330014449 5360; E-mail: isabelle.desguerre@nck.aphp.fr
}

Received 5 March 2012; revised 30 August 2012; accepted 25 September 2012; published online 9 January 2013 
question of a correlation between dystrophin expression and clinical weakness remains debatable. ${ }^{9,17}$ In the specific situation of balanced $\mathrm{X}$ chromosome-autosome translocations disrupting the dystrophin gene, completely skewed inactivation of the non-translocated X chromosome leads to inactivation of the second $D M D$ allele and therefore to a clinical phenotype as severe as in boys with DMD. ${ }^{18}$ However, whereas it is widely accepted that X-chromosome inactivation (XCI) has a role in the clinical variability in DMD carriers, $\mathrm{XCI}$ seems to be insufficient to predict the phenotypic status and degree of muscle weakness in young DMD carriers. ${ }^{19,20}$

To better understand the mechanisms of this variability, we reviewed clinical, histological and genetic parameters including XCI pattern of 26 early pediatric symptomatic DMD carriers.

\section{MATERIALS AND METHODS}

Patients clinical parameters and molecular diagnosis

In this study, 26 female patients referred over the past 10 years to several French neuromuscular centers were included on the following criteria: (i) the identification of a mutation in the DMD gene and (ii) the onset of manifesting symptoms before 17 years. Data were collected from completed information sheets as shown in Table 1. Duchenne-like phenotype was considered when patients lost ambulation before the age of 15 years or were supposed to for patients younger than 15 years at the time of the study. Muscle weakness with persistence of ambulation after 15 years was considered as Becker-like phenotype. As muscle testing was not performed for most carriers, the severity of the muscle weakness has been estimated on the global clinical and functional evaluation. Patients were considered to have cardiac dysfunction with left ventricular ejection fraction $\leqslant 50 \%$. Exhaustive clinical data were not available for all cases particularly for the oldest patients. Cognitive status was evaluated by both educational level and general intelligence assessment scale (Wechsler Intelligence Scale for Children, WISC III) with intellectual deficiency defined by IQ $<70 .^{21,22}$ Two patients (\#6 and \#13) had been previously published. ${ }^{18,23} D M D$ gene analyses were performed in three French hospital laboratories (Cochin hospital, Paris; CHU, Tours; CHU, Rennes). Different methods, including multiplex PCR reactions, MLPA and/or mRNA analysis by RT-PCR and cDNA direct sequencing, were used to identify $D M D$ gene mutations. All samples and clinical data were collected after informed consent had been obtained.

\section{Histological analysis of the muscle}

Muscle biopsy and histomorphological study, as well as muscle proteins analysis by immuno-histochemistry and multiplex western blot (WB) were performed for 20 patients. ${ }^{24}$

\section{Genetic analyses}

XCI studies were performed by analysis of the polymorphic CAG repeat in $A R$ gene $^{25}$ on DNA extracted from peripheral blood and/or muscle biopsies. The degree of XCI skewing was expressed as the ratio of one allele expression versus the other. The XCI pattern could not be determined in patients who were non informative (homozygous at the CAG repeat) for the CAG repeat in $A R$ gene.

Karyotype analysis was performed to exclude cytogenetic abnormalities that could lead to a skewed XCI pattern. For patients with cognitive impairment, CGH array and Fragile X syndrome gene analysis were performed.

\section{Statistical analysis}

Fisher's exact test was used for statistical analyses with a type I error of 0.05 , by convention.

\section{RESULTS}

The clinical and molecular data are summarized in (Table 1). The first symptoms in childhood were muscular weakness, limb girdle deficit as assessed by clinical examination or muscular manual testing $(n=11)$, abnormal gait $(n=10)$, exercise intolerance $(n=8)$ and/or myalgia $(n=10)$. The age of first symptoms was before 6 years $(n=8)$, between $6-12$ years $(n=16)$ and $12-15$ years $(n=2)$. In one case, the disease was revealed by a rhabdomyolysis mimicking a metabolic myopathy. Clinical severity consisted of Duchenne-like phenotype $(n=5)$, Becker-like phenotype $(n=13)$ or exercise intolerance $(n=8)$. Muscle weakness was predominant in the lower limb girdle $(n=22)$, severe $(n=9)$, moderate $(n=13)$ and rarely asymmetrical $(n=2)$. Five patients had lost ambulation after 40 years and two before 15 years (three other DMD-like carriers were still under 10 years and had a delay in walking with abnormal gait and a severe muscle weakness). The SCK levels were always increased ranging from $330 \mathrm{UI} / 1$ to $34400 \mathrm{UI} / \mathrm{l}$. Cardiac dysfunction was present in 19\% (two cases during childhood without heart symptoms) and three cases after 30 years (including two cases of severe cardiomyopathy). Cognitive impairment was found in $27 \%$ (7/26), two with intellectual disability confirmed by IQ test (VIQ and PIQ, respectively) and five with learning disabilities (specialized schooling or with a delay of more than two years in schooling). The 19 remaining patients had normal or subnormal educational level.

A total of 23 muscle biopsies were performed with age ranking from 4-60 years and classical pathological analysis was available in 18 cases: muscular dystrophy $(n=15)$ or non-specific myopathic changes $(n=3)$ (Figure 1). The muscle biopsies performed before the age of 6 years ( 8 cases) revealed a severe muscular dystrophy with endomysial fibrosis (carriers $\# 2, \# 3, \# 4, \# 5, \# 8$ ) correlated with the severity of the muscular phenotype in five cases. In the three remaining cases, we observed irregular size of the muscle fibers, rare focal fiber necrosis correlated with exercise intolerance and a moderate muscle deficit. In the other cases (10 cases), the muscular biopsies confirmed a dystrophic pattern but were performed around 12 years of age (4 cases) or after 40 years ( 6 cases) after a long time of clinical evolution.

Immunostaining analysis using antibodies against dystrophin was abnormal in all tested cases (21 cases). A mosaic pattern was observed in 17 cases, a diffuse irregular staining in 3 cases and lack of staining in 1 case. The WB analysis was performed in 19 patients and was abnormal in all cases with lack of dystrophin $(n=1)$, decreased amount of normal molecular weight dystrophin $(n=15)$ or decreased amount of truncated dystrophin $(n=3)$ (Figure 2)). No precise quantification of dystrophin could be performed, but the patient with complete lack of dystrophin had the most severe muscular impairment.

As specified in inclusion criteria, all the 26 patients included in this study had an identified $D M D$ gene mutation. Family history of dystrophinopathy was found in 13/26 cases. Parents' molecular data were available in 23 families and showed that $D M D$ gene mutations had occurred de novo in 35\% (8/23) and were inherited in 65\% (15/23).

DMD gene large deletions were found in $54 \%$ of the cases $(14 / 26)$ (Figure 3). All of them were out of frame leading to a premature stop codon and 79\% (11/14) were located between exons 44 and 55 within the distal hot spot of rearrangements of the gene. Four large duplications and one large triplication were observed (19\%), and most of them (4/5) were predicted to disrupt the reading frame. Point mutations were identified in $27 \%$ (7/26): nonsense (4/26), frameshift $(2 / 26)$ and splicing mutations (1/26).

Learning disabilities assessed through scholar and education performances were diagnosed in five patients. For two others patients, diagnosis of intellectual disability was based on WISC III evaluation (VIQ:71, PIQ:58 for carrier \#1 and VIQ:61; PIQ:71 for carrier \#3). No autism spectrum disorder was noticed. As expected, in this group of patients, we found a high percentage $(86 \%)$ of DMD mutations involving Dp71 $(n=2)$ or Dp140 $(n=4)$ isoforms. Indeed, these mutations include deletions of exons $51-53$ and of exons 28-54, encompassing the first coding exon of Dp140 isoform (exon 51). ${ }^{26}$ For Dp71 whose coding sequence starts at the exon 63 , the mutations 


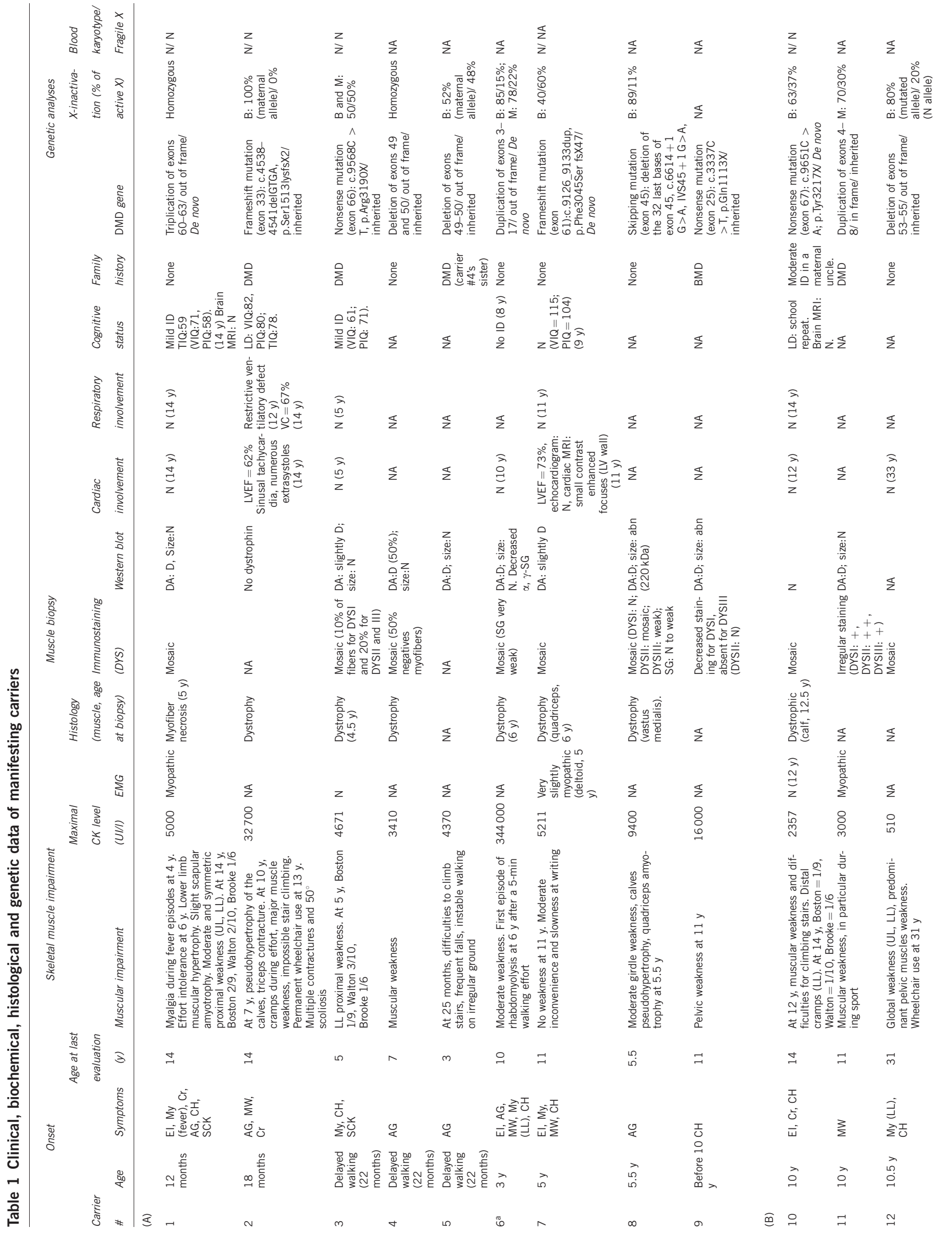




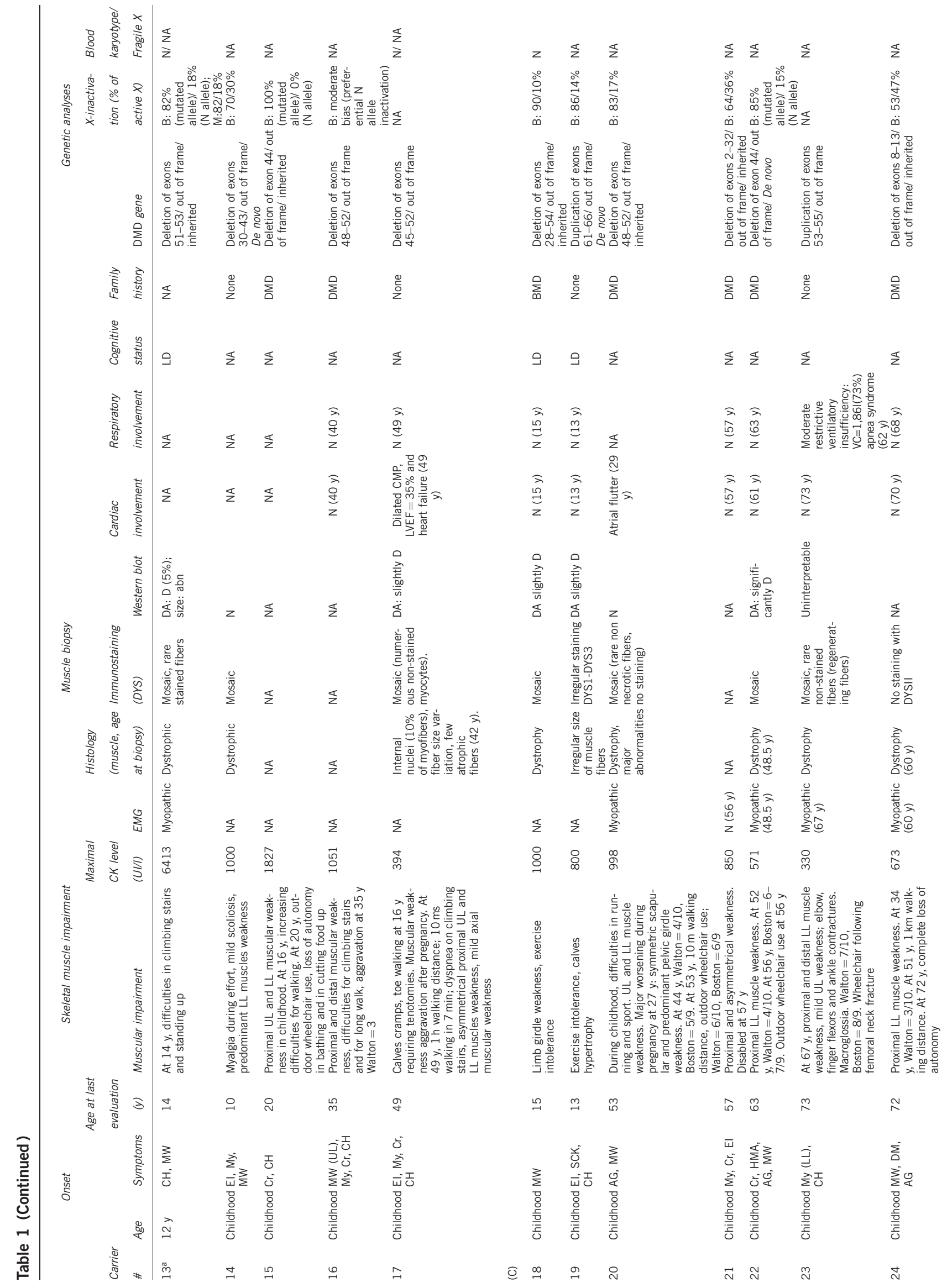




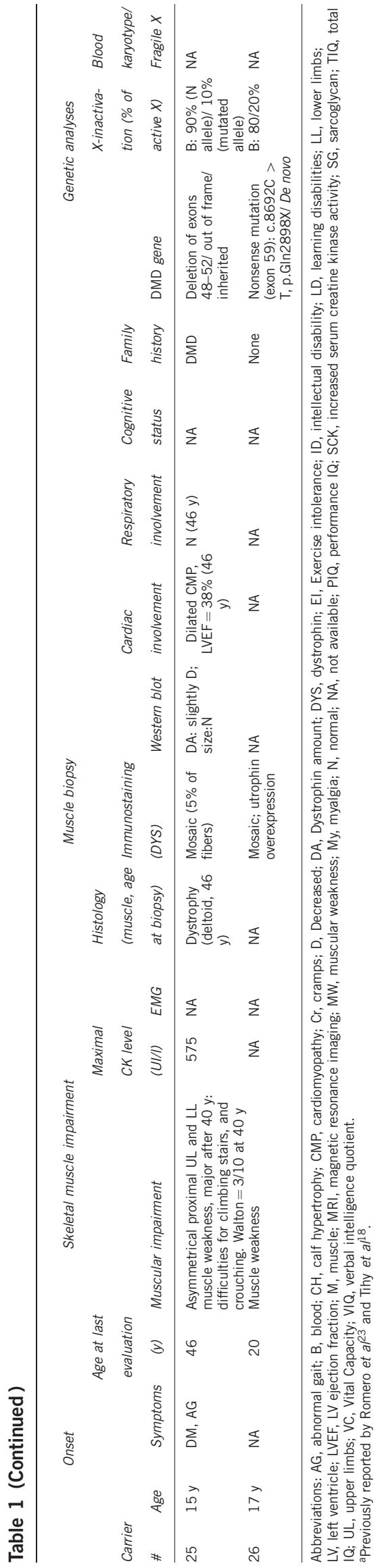

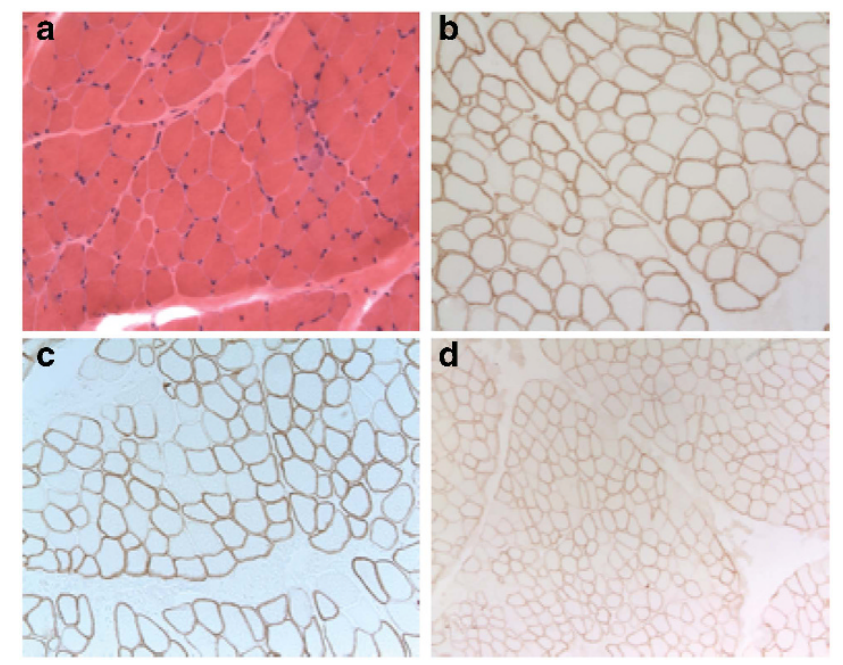

Figure 1 Histological findings and immunostaining on a DMD carrier muscle biopsy (patient \#3). (a) Dystrophic aspect with fiber size variation (Hemateineosine staining, $\times 200$ ). (b, c and d) Mosaic aspect of dystrophin staining with DYS1 $(\mathbf{b}, \times 200)$, DYS2 $(\mathbf{c}, \times 200)$ and DYS3 $(\mathbf{d}, \times 100)$ antibodies, respectively, targettting dystrophin rod, C-terminal and $\mathrm{N}$-terminal domains.

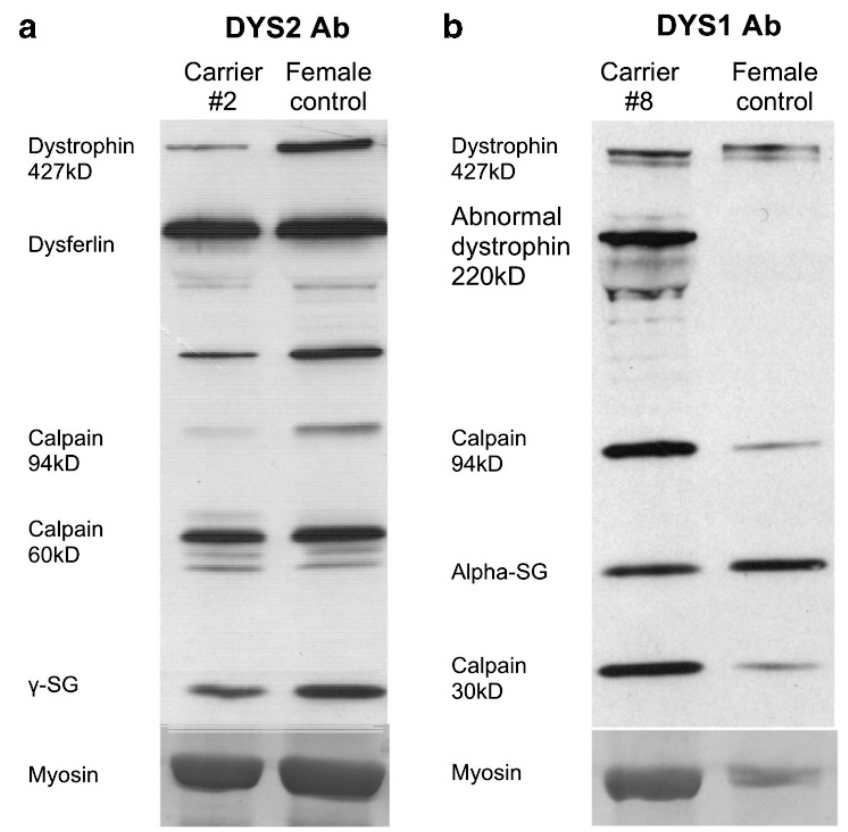

Figure 2 Dystrophin analysis by multiplex western blot revealing decreased amount of dystrophin compared with the control. (a) DYS2 antibody: normal molecular weight (MW) (carrier \#2); (b) DYS1 antibody: abnormal MW with an additional band of $220 \mathrm{kD}$ (carrier \#8). Dysferlin, calpain, $\alpha$ - and $\gamma$-sarcoglycan protein bands are also displayed.

were nonsense mutations in exons 66 and 67, respectively. In the two other patients, DMD abnormalities correspond to one duplication of exons 61-66 and one triplication of exons 60-63 predicted to disrupt all DMD products, including Dp140 and Dp71 isoforms.

XCI patterns were determined on blood DNA for 21 informative patients and on muscle DNA for only 4 patients because of the restricted availability of this tissue. XCI was skewed in $62 \%$ of cases on blood DNA: $10 \%$ with a completely skewed pattern (100:0) and 52\% with a significantly skewed pattern $(\geqslant 80: 20)$ (Figure 4$)$. In the seven 


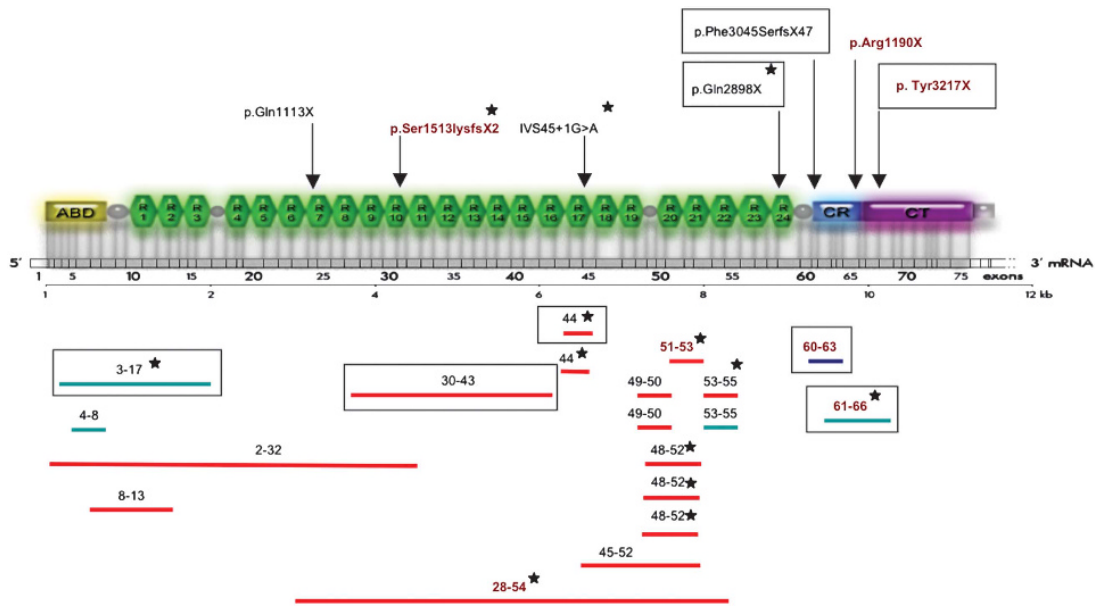

Figure 3 Schematic representation of the DMD gene mutations detected in the 26 patients. Inheritance type, $\mathrm{XCl}$ pattern and cognitive impairment are indicated. Carrier number (Table 1) is represented in blue in brackets; cognitive impairment in red; DMD gene deletions, duplication, triplication, respectively, in red, green and blue lines (Numbers associated with deletions and duplications correspond to deletions and duplications boundaries); skewed $\mathrm{XCl}$ is indicated by a star and de novo occurrence by a box.

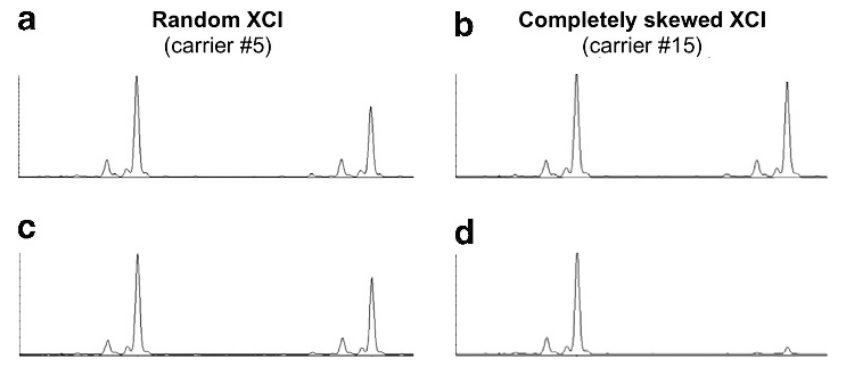

Figure 4 X-inactivation patterns in DMD carriers. AR locus, Xq11-q12: automatic sequencer traces correspond to the PCR products of undigested DNA (a, c) and after Hpall digestion (b, d). (a, b) Random X-inactivation pattern (52:48) in patient \#5. (c, d) Completely skewed X-inactivation pattern (100:0) in patient \#15.

patients where the two alleles could be distinguished, the normal allele was preferentially inactivated in all cases but one who was analyzed at the age of 40 years. On muscle DNA, XCI was skewed in one case and a nearly significant pattern (78:22) was observed in another patient. XCI was random in two patients although they had significant muscle weakness before 10 years. In three patients, we found the same XCI patterns when determined simultaneously on blood and on muscle.

Among patients with skewed XCI, the ratio of deletions and duplications versus point mutations was not significantly different from the entire series ( $67 \%$ versus $73 \%$ for deletions; $30 \%$ versus $27 \%$ for point mutations). In addition, $31 \%$ of the patients with skewed $\mathrm{XCI}$ had de novo mutations (one deletion, two duplications and one point mutation). This was similar to the ratio of the entire series (35\%). Finally, $67 \%$ of the seven patients with cognitive impairment had a skewed XCI including one with a completely skewed pattern.

\section{DISCUSSION}

We report the largest series of DMD female carriers symptomatic in childhood (Table 2). In the two largest reported series of DMD carriers, the proportion of manifesting carriers varied from 5 to $22 \%$ and the pediatric cases remained rare. ${ }^{4,20}$

In our patients, like in the other pediatric series, hyperCKemia was constant. ${ }^{27-30}$ Muscle weakness was also prevalent and found in $88 \%$ of cases. Exercise intolerance was the first symptom in $27 \%$ and remained the only muscular symptom during childhood in three cases, including one case with acute rhabdomyolysis episodes mimicking a metabolic myopathy.

The clinical presentation of our patients fit within three clinical phenotypes: Duchenne-like clinical spectrum ( 5 cases, among them 2 were wheelchair bounded before 15 years), Becker-like clinical spectrum (13 cases, 5 wheelchair bounded after 40 years) and early exercise intolerance with mild muscular deficit at adult age (8 cases).

Cardiac involvement was observed in $19 \%$ of our patients independently of the severity of muscle involvement and the age of the first symptoms. In large series, cardiac abnormalities on echocardiogram were identified in $23-38 \%$ of cases and dilated cardiomyopathies in $8-18 \%$. ${ }^{4,6}$ Our data confirm that cardiac dysfunction in DMD carriers usually occurs most of the time after 30 years but need to be searched for during childhood in early symptomatic carriers (two cases before 15 years).

In previous series of DMD female cases, cognitive impairment is not frequently reported except for the cases with chromosomal abnormalities. ${ }^{15,28-30}$ In our study, we found that 7 out of our 26 female carriers had cognitive impairment, 2 with intellectual disability and 5 with learning disabilities thus highlighting the high rate $(27 \%)$ of cognitive impairment in manifesting carriers. The prevalence of intellectual disability is estimated at $\sim 2-3 \%$ in the general population and males are more often affected than females. ${ }^{31}$ Considering a prevalence of $3 \%$, which is likely to be an overestimation in female population, we found a significantly higher proportion of carriers with cognitive impairment in our series versus in the general population $(P=0.002)$.

\section{Muscle study and protein expression in the muscle}

The five most severe cases have also the early dystrophic pattern with endomysial fibrosis at 5 years in accordance with the histological data known in Duchenne boys. ${ }^{2}$ Manifesting female carriers with muscle weakness usually have a mosaic pattern of dystrophin in muscle shown by immunostaining. This mosaicism is believed to result from the formation of multinucleate muscle fibers from fusion of uninucleate myoblasts with different dystrophin expression based on different XCI. Abnormal qualitative and/or quantitative WB patterns can also be helpful for the diagnosis in female patients with less evocative clinical picture. The muscle biopsy was critical for the diagnosis, particularly in the case with pseudometabolic 


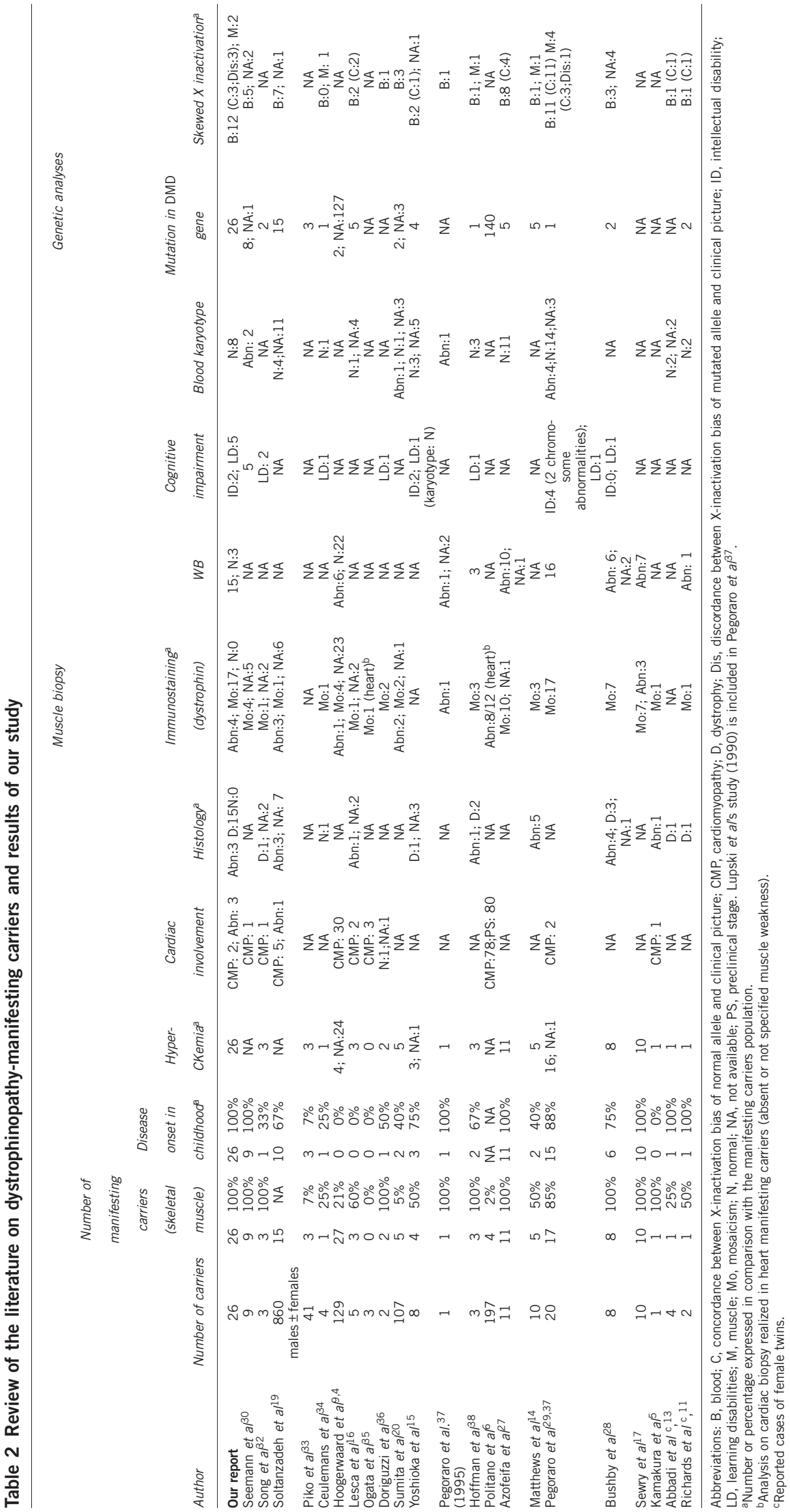


presentation. In this study, muscle biopsy analysis was contributory in $100 \%$ of cases either on histological aspect of dystrophy, mosaic immunostaining and/or abnormal WB. Surprisingly, Hoogerwaard et al ${ }^{9}$ performed 50 muscle biopsies in DMD carriers and reported immunohistopathological abnormalities in a low proportion of cases ( $10 \%$ of dystrophin mosaicism and abnormal WB), probably because this study was carried out in asymptomatic $(n=23)$ or symptomatic carriers in adulthood $(n=27)$, and based on needle biopsies.

\section{The dystrophin gene and the transcripts}

Not surprisingly, in our group of symptomatic patients in childhood, all the mutations are predicted to disrupt DMD gene structure and lead to loss of function of dystrophin. Complete study of the dystrophin gene is lacking in the oldest series of DMD carriers. ${ }^{4,28}$ Recently, Soltanzadeh et al ${ }^{19}$ reviewed the clinical and genetic features in 15 manifesting carriers identified among 860 subjects within the United Dystrophinopathy Project. They found $48 \%$ of exonic deletions or duplications, whereas in our study large rearrangements represent $73 \%$ of the mutations, and $52 \%$ of point mutations versus $27 \%$ in our patients. The distribution of the mutations in these two series is not significantly different $(P=0.18)$, although the small numbers in the two groups impair precise statistical analysis.

Compared with the large French UMD-DMD database (2405 patients), 54\% of $D M D$ gene deletions in our study are not significantly different from the $61 \%$ reported in DMD patients $(P=0.55) .{ }^{39} \mathrm{In}$ all, $79 \%$ of these deletions start in the distal hot spot (exons $44-55$ ), as observed in the UMD-DMD database (74\%). We report a similar proportion of duplications (15\% of our carriers versus $13 \%$ in DMD patients) and of point mutations (27\% in our series versus $26 \%)^{24,40,41}$ In addition, the rate of de novo occurrence of the mutations in our series $(35 \%)$ is not significantly different from the rate reported in the UMD-DMD database $(24.5 \%)(P=0.37)$.

Point mutations occurred de novo in $37.5 \%$ of our series versus $19.7 \%$ in the UMD-DMD database but this difference was not significant maybe because of the relatively small number of cases $(P=0.42)$. Interestingly, we found a significant difference concerning the occurrence of de novo deletions, which represent $25 \%$ of the de novo mutations versus $71 \%$ in the UMD-DMD database $(P=0.01){ }^{39}$ Even if we do not know the parental origin of the de novo events, this lower proportion of de novo deletions compared with de novo point mutations in female carriers could be explained by the possible paternal origin of new mutations, which cannot be observed in DMD boys.

In addition, we noticed that the majority of the duplications or triplication were associated with severe muscular phenotype in $4 / 5$ patients $(80 \%)$. Cognitive impairment was observed in two patients with a duplication of exons 61-66 and one with a triplication of exons 60-63. The size of the series is not large enough to conclude to a significant link between duplications/triplications and a more severe phenotype but this data seems to be relevant and needs to be confirmed.

\section{$\mathrm{X}$-inactivation pattern in lymphocyte and muscle DNA}

XCI pattern was skewed toward nonrandom pattern in $62 \%(13 / 21)$ of informative carriers on blood and/or muscle. This percentage is significantly higher than reported by Sumita et al ${ }^{20}$ in asymptomatic carriers $(37 \%)(P<0.05)$, but comparable to the study of Soltanzadeh et $a l^{19}$ in pediatric manifesting carriers. We suggest that this high proportion of skewed XCI is related to the young age and the clinical expression in the female carriers.

Indeed, our population of carriers corresponds to severe phenotypes with onset of symptoms in childhood. As expected, we found a higher rate of skewed XCI than in studies concerning less symptomatic or even asymptomatic carriers. However, we did not observed XCI bias in every carrier of our series. For example, as expected, completely skewed XCI was found in two carriers with very severe phenotype (Table $1 \mathrm{~A}$ and B; carriers \#2 and \#15). Conversely, XCI pattern was random in three other carriers with onset of symptoms before 10 years (Table $1 \mathrm{~A}$, carriers \#3, \#5 and \#7).

To further analyze the role of $\mathrm{X}$ inactivation in the phenotype, we assessed whether XCI pattern depends on the type of mutation. Overall, skewed XCI was observed in 10/15 deletions or duplications and 3/6 point mutations, that is, two nonsense mutations and one splicing mutation, which is in agreement with mutation type distribution in our series. In the two carriers with completely skewed XCI, the DMD mutations were inherited and there was no family history of XCI bias. Like Soltanzadeh et $a l^{19}$, we found a predominantly skewed XCI in deletions and duplications: the frequency was even higher in our series, $67 \%$ versus $57 \%$ in Soltanzadeh's series. On the opposite, we report skewed XCI in $50 \%$ of point mutations, whereas Soltanzadeh et al ${ }^{19}$ did not find any bias in the six carriers of their study. Using Fisher's test analysis, we did not observe a significant difference between rates of skewed XCI patterns in rearrangements and in point mutations $(P=0.63)$. The normal allele was preferentially inactivated in all carriers except the carrier investigated at 40 years. Interestingly, we found the same XCI patterns in lymphocyte and muscle DNA, but these analyses could be simultaneously performed in only three patients, which is insufficient to establish a correlation between blood and muscle XCI patterns. Azofeifa et a ${ }^{27}$ found a significant statistical correlation between lymphocyte and muscle XCI ratios, whereas Matthews et al ${ }^{14}$ showed differences between XCI patterns in muscle and in other tissues, even of same embryonic origin. Furthermore, XCI pattern may be modified in multinucleate muscle fibers compared with single nucleate lymphocytes. ${ }^{37}$ Indeed, Pegoraro et $a l^{37}$ proposed a model of biochemical normalization in random inactivation patients and genetic normalization in skewed $\mathrm{X}$-inactivation carriers. This could explain the preferential normal expressed allele in patient \#25 explored at 46 years, but this model cannot be applied to the $38 \%$ of manifesting carriers with random XCI in our cohort. Other mechanisms are certainly involved to explain the severity of the phenotype.

Finally, we focus on cognitive impairment in the seven manifesting carriers. All the female patients with cognitive impairment except one $(86 \%)$ had a mutation in the end part of the dystrophin gene, involving Dp140 or Dp71 as previously reported in several series of DMD male patients. ${ }^{26,42,43}$ These findings therefore provide additional arguments in favor of the crucial role of Dp71 and Dp140 in the development of cognitive function.

Cognitive impairment observed in 2 of the 12 patients with mutations implicating Dp140 had skewed XCI pattern. Four females had mutations implicating Dp71 with skewed XCI pattern observed in $2 / 3$ cases (case \#1 was uninterpretable). These findings clearly support that manifesting carriers with mutation in Dp71 are more prone to have cognitive impairment like DMD male patients. Seemann et $a l^{30}$ recently reported 5/9 cases of dystrophinopathies in female children with learning disability: a translocation was present in two cases and skewed XCI pattern in one case but no correlation with the location of the mutation at the end of the gene was highlighted. ${ }^{44}$

To explain occurrence of cognitive deficit in females, we propose a cell-nonautonomous effect by which function of cells expressing the normal $D M D$ allele could be negatively influenced by the phenotype of cells bearing the mutated DMD gene. ${ }^{44}$ This mechanism could underly brain and neuronal dysfunction through deregulation of neuronal connectivity mediated by cumulative loss of function of 
$D M D$ gene products, including Dp71. One can also wonder to what extent this mechanism could affect homeostasis of muscle fibers and provide insights to understand muscle phenotypic heterogeneity.

In conclusion, our series consists of the largest series of manifesting DMD female carriers in childhood reported to date and widely confirms other works. However, this study focuses on specific findings such as the frequency of exercise intolerance and cognitive impairment. On the one hand, we have shown that exercise intolerance or pseudometabolic symptoms are a common presentation in these carriers with early onset of symptoms. On the other hand, we recommend a careful evaluation of intellectual functioning, as cognitive impairment is frequently associated (27\%). Furthermore, our results highlight a phenotype-genotype correlation for cognitive impairment, which is particularly found when the mutation involves Dp140 and above all Dp71 isoforms. In view of our data on XCI pattern, we propose that significant skewed XCI pattern found in $62 \%$ of the carriers explains the severe phenotype in our series and that it seems to be a good correlation of XCI pattern on muscle and on lymphocytes when studied at a young age. However, XCI pattern is not sufficient to predict the clinical picture as shown in 38\% of the manifesting carriers with random XCI. Further studies are required to better understand physiopathological mechanisms involved in manifesting DMD carriers and provide a comprehensive view of factors influencing natural history and severity of the phenotype to address critical issues raised in the context of genetic counseling.

\section{CONFLICT OF INTEREST}

All the authors declare no potential conflict of interest except Dr Urtizberea who has received compensation for consultancy and development of educational presentations and Dr Gherardi who has received unrelated basic research grants from the Association Française contre les Myopathies (AFM).

\section{ACKNOWLEDGEMENTS}

We gratefully acknowledge the patients and families for their participation in the study and the physicians who referred them. We particularly thank MariePierre Moizard (CHU, Tours), Jennifer Chauvel-Breton (CHU, Rennes), Caroline Beugnet and all members of the Laboratoire de Biochimie Génétique et Moléculaire and the Cell Bank-Cochin APHP for their help and advice.

1 Emery AE: The muscular dystrophies. Lancet 2002; 359: 687-695

2 Desguerre I, Christov C, Mayer M et al: Clinical heterogeneity of duchenne muscular dystrophy (DMD): definition of sub-phenotypes and predictive criteria by long-term follow-up. PLoS One 2009; 4: e4347.

3 Pegoraro E, Hoffman EP, Piva L et al: SPP1 genotype is a determinant of disease severity in Duchenne muscular dystrophy. Neurology 2011; 76: 219-226.

4 Hoogerwaard EM, van der Wouw PA, Wilde AA et al: Cardiac involvement in carriers of Duchenne and Becker muscular dystrophy. Neuromuscul Disord 1999; 9: 347-351.

5 Kamakura K, Kawai M, Arahata K, Koizumi H, Watanabe K, Sugita H: A manifesting carrier of Duchenne muscular dystrophy with severe myocardial symptoms. J Neurol 1990; 237: 483-485.

6 Politano L, Nigro V, Nigro G et al: Development of cardiomyopathy in female carriers of Duchenne and Becker muscular dystrophies. JAMA 1996; 275: 1335-1338.

7 Nolan MA, Jones OD, Pedersen RL, Johnston HM: Cardiac assessment in childhood carriers of Duchenne and Becker muscular dystrophies. Neuromuscul Disord 2003; 13: 129-132.

8 Grain L, Cortina-Borja M, Forfar C, Hilton-Jones D, Hopkin J, Burch M: Cardiac abnormalities and skeletal muscle weakness in carriers of Duchenne and Becker muscular dystrophies and controls. Neuromuscul Disord 2001; 11: 186-191.

9 Hoogerwaard EM, Ginjaar IB, Bakker E, de Visser M: Dystrophin analysis in carriers of Duchenne and Becker muscular dystrophy. Neurology 2005; 65: 1984-1986.

10 Saillour Y, Cossée M, Leturcq F et al: Detection of exonic copy-number changes using a highly efficient oligonucleotide-based comparative genomic hybridization-array method. Hum Mutat 2008; 29: 1083-1090.

11 Richards CS, Watkins SC, Hoffman EP et al: Skewed X inactivation in a female MZ twin results in Duchenne muscular dystrophy. Am J Hum Genet 1990; 46: 672-681.

12 Lupski JR, Garcia CA, Zoghbi HY, Hoffman EP, Fenwick RG: Discordance of muscular dystrophy in monozygotic female twins: evidence supporting asymmetric splitting of the inner cell mass in a manifesting carrier of Duchenne dystrophy. Am J Med Genet 1991; 40: 354-364

13 Abbadi $N$, Philippe $C$, Chery $M$ et al: Additional case of female monozygotic twins discordant for the clinical manifestations of Duchenne muscular dystrophy due to opposite X-chromosome inactivation. Am J Med Genet 1994; 52: 198-206.

14 Matthews PM, Benjamin D, Van Bakel I et al: Muscle X-inactivation patterns and dystrophin expression in Duchenne muscular dystrophy carriers. Neuromuscul Disord 1995; 5: 209-220.

15 Yoshioka M, Yorifuji T, Mituyoshi I: Skewed X inactivation in manifesting carriers of Duchenne muscular dystrophy. Clin Genet 1998; 53: 102-107.

16 Lesca G, Demarquay G, Llense S et al: [Symptomatic carriers of dystrophinopathy with chromosome X inactivation bias]. Rev Neurol (Paris) 2003; 159: 775-780.

17 Sewry CA, Sansome A, Clerk A et al: Manifesting carriers of Xp21 muscular dystrophy; lack of correlation between dystrophin expression and clinical weakness. Neuromuscul Disord 1993; 3: 141-148.

18 Tihy F, Vogt N, Recan D et al: Skewed inactivation of an X chromosome deleted at the dystrophin gene in an asymptomatic mother and her affected daughter. Hum Genet 1994; 93: 563-567.

19 Soltanzadeh P, Friez MJ, Dunn D et al: Clinical and genetic characterization of manifesting carriers of DMD mutations. Neuromuscul Disord 2010; 20: 499-504.

20 Sumita DR, Vainzof M, Campiotto $S$ et al: Absence of correlation between skewed $X$ inactivation in blood and serum creatine-kinase levels in Duchenne/Becker female carriers. Am J Med Genet 1998; 80: 356-361.

21 Wechsler D: WPPSI-R Manual: Wechsler Preschool and Primary Scale of IntelligenceRevised 1989.

22 Wechsler D: WISC III Manual: Wechsler Scale Intelligence for Children 1991.

23 Romero NB, De Lonlay P, Llense S et al: Pseudo-metabolic presentation in a Duchenne muscular dystrophy symptomatic carrier with 'de novo' duplication of dystrophin gene. Neuromuscul Disord 2001; 11: 494-498.

24 Deburgrave N, Daoud F, Llense S et al: Protein- and mRNA-based phenotype-genotype correlations in DMD/BMD with point mutations and molecular basis for BMD with nonsense and frameshift mutations in the DMD gene. Hum Mutat 2007; 28: 183-195.

25 Allen RC, Zoghbi HY, Moseley AB, Rosenblatt HM, Belmont JW: Methylation of Hpall and Hhal sites near the polymorphic CAG repeat in the human androgen-receptor gene correlates with X chromosome inactivation. Am J Hum Genet 1992; 51: 1229-1239.

26 Taylor PJ, Betts GA, Maroulis S et al: Dystrophin gene mutation location and the risk of cognitive impairment in Duchenne muscular dystrophy. PLoS One 2010; 5: e8803.

27 Azofeifa J, Voit T, Hubner C, Cremer M: X-chromosome methylation in manifesting and healthy carriers of dystrophinopathies: concordance of activation ratios among first degree female relatives and skewed inactivation as cause of the affected phenotypes. Hum Genet 1995; 96: 167-176.

28 Bushby KM, Goodship JA, Nicholson LV, Johnson MA, Haggerty ID, Gardner-Medwin D: Variability in clinical, genetic and protein abnormalities in manifesting carriers of Duchenne and Becker muscular dystrophy. Neuromuscul Disord 1993; 3: 57-64.

29 Pegoraro E, Schimke RN, Arahata K et al: Detection of new paternal dystrophin gene mutations in isolated cases of dystrophinopathy in females. Am J Hum Genet 1994; 54: 989-1003.

30 Seemann N, Selby K, McAdam L et al: Symptomatic dystrophinopathies in female children. Neuromuscul Disord 2011; 21: 172-177.

31 Leonard H, Wen X: The epidemiology of mental retardation: challenges and opportunities in the new millennium. Ment Retard Dev Disabil Res Rev 2002; 8: 117-134.

32 Song TJ, Lee KA, Kang SW, Cho H, Choi YC: Three cases of manifesting female carriers in patients with Duchenne muscular dystrophy. Yonsei Med J 2011; 52: 192-5.

33 Pikó H, Vancsó V, Nagy B, Bán Z, Herczegfalvi A, Karcagi V: Dystrophin gene analysis in Hungarian Duchenne/Becker muscular dystrophy families - detection of carrier status in symptomatic and asymptomatic female relatives. Neuromuscul Disord 2009; 19: 108-12.

34 Ceulemans BP, Storm K, Reyniers Jr. E et al: Muscle pain as the only presenting symptom in a girl with dystrophinopathy. Pediatr Neurol 2008; 38: 64-6.

35 Ogata $\mathrm{H}$, Nakagawa $\mathrm{H}$, Hamabe $\mathrm{K}$ et al: A female carrier of Duchenne muscular dystrophy complicated with cardiomyopathy. Intern Med 2000; 39: 34-8.

36 Doriguzzi C, Palmucci L, Mongini T et al: Variable histological expression of dystrophinopathy in two females. Acta Neuropathol 1999; 97: 657-60.

37 Pegoraro E, Schimke RN, Garcia C et al: Genetic and biochemical normalization in female carriers of Duchenne muscular dystrophy: evidence for failure of dystrophin production in dystrophin-competent myonuclei. Neurology 1995; 45: 677-690.

38 Hoffman EP, Pegoraro E, Scacheri P et al: Genetic counseling of isolated carriers of Duchenne muscular dystrophy. Am J Med Genet 1996; 63: 573-80.

39 Tuffery-Giraud S, Beroud C, Leturcq F et al: Genotype-phenotype analysis in 2,405 patients with a dystrophinopathy using the UMD-DMD database: a model of nationwide knowledgebase. Hum Mutat 2009; 30: 934-945.

40 White $\mathrm{S}$, Kalf M, Liu $\mathrm{Q}$ et al: Comprehensive detection of genomic duplications and deletions in the DMD gene, by use of multiplex amplifiable probe hybridization. Am J Hum Genet 2002; 71: 365-374.

41 Zeng F, Ren ZR, Huang SZ et al: Array-MLPA: comprehensive detection of deletions and duplications and its application to DMD patients. Hum Mutat 2008; 29: 190-197.

42 Daoud F, Angeard N, Demerre B et al: Analysis of Dp71 contribution in the severity of mental retardation through comparison of Duchenne and Becker patients differing by mutation consequences on Dp71 expression. Hum Mol Genet 2009; 18: 3779-3794.

43 Moizard MP, Toutain A, Fournier D et al: Severe cognitive impairment in DMD: obvious clinical indication for Dp71 isoform point mutation screening. Eur J Hum Genet 2000; 8: 552-556.

44 Apfeld J, Kenyon C: Cell nonautonomy of C. elegans daf-2 function in the regulation of diapause and life span. Cell 1998; 95: 199-210. 